\title{
Síndrome de Lyell causado por el uso concomitante de carbamazepina y ciprofloxacino. Presentación de un caso
}

\section{Lyell syndrome caused by the concomitant use of carbamazepine and ciprofloxacin. Case presentation}

\author{
Angélica Ma. Hernández-Fernández ${ }^{1,2 *}$, José A. Flores-Vargas ${ }^{1,2}$ y Lidia A. Cabrera-Velazquez ${ }^{2}$ \\ ${ }^{1}$ Centro Integral de Servicios Farmacéuticos; ${ }^{2}$ Centro Institucional de Farmacovigilancia. Hospital Regional de Alta Especialidad de Ixtapaluca, Ixtapaluca, Edo. Méx. México
}

\begin{abstract}
Toxic epidermal necrolysis (TEN) or Lyell syndrome is a severe mucocutaneous reaction characterized by inappropriate immune response mediated by lymphocytes to certain drugs or their metabolites. The mortality rate for TEN is 25-30\%. TEN begins as a maculo-papular rash that evolves into ampules formation, with epidermis necrosis and its detachment; among the main clinical complications are damage to the respiratory and gastrointestinal mucosae, electrolytic imbalance, kidney damage and multi-organ failure. Here, we present a case of a female patient diagnosed with TEN due to the concomitant use of ciprofloxacin and carbamazepine, who presented with severe electrolytic imbalance, mainly hypocalcemia, with a compromised cardiac function. The patient was treated with immunoglobulin $\mathrm{G}$ and corticosteroid, and had a satisfactory recovery.
\end{abstract}

Key words: Ciprofloxacin. Carbamazepine. Macules. Toxic epidermal necrolysis.

\section{Resumen}

La necrólisis epidérmica tóxica o síndrome de Lyell es una reacción mucocutánea grave, caracterizada por una activación inmunitaria inapropiada en respuesta a ciertos medicamentos o sus metabolitos mediada por linfocitos, y tiene una mortalidad del 25-30\%. Inicia como un exantema maculopapular que evoluciona a la formación de ámpulas, necrosis de la epidermis y desprendimiento de esta. Entre sus principales complicaciones se encuentran: afectación de mucosas respiratorias y gastrointestinales, descompensación electrolítica, lesión renal y falla multiorgánica. Se presenta el caso de una paciente con diagnóstico de necrólisis epidérmica tóxica debido al uso concomitante de ciprofloxacino y carbamazepina, presentando un cuadro agravado con descompensación electrolítica, principalmente hipocalcemia, comprometiendo la función cardiaca. La paciente fue manejada con inmunoglobulina G y corticosteroide, logrando una recuperación satisfactoria.

Palabras clave: Ciprofloxacino. Carbamazepina. Máculas. Necrólisis epidérmica tóxica.

\section{Correspondence:}

*Angélica Ma. Hernández-Fernández

E-mail: anglauh@hotmail.com
Date of reception: $24-10-2019$

Date of acceptance: 09-06-2020

DOI: $10.24875 /$ HMCM.20000243
Available online: 09-07-2020 Hosp Med Clin Manag. 2020;13:77-81

2604-0018 / @ 2020 Mexican Regional Hospitals of High Specialty and Federal Hospitals. Published by Permanyer. This is an open access article under the CC BY-NC-ND license (http://creativecommons.org/licenses/by-nc-nd/4.0/). 


\section{INTRODUCCIÓN}

La necrólisis epidérmica tóxica (NET), también conocida como síndrome de Lyell, fue descrita en 1956 como una enfermedad parecida a las escaldaduras. Es una reacción cutánea adversa grave, que inicialmente era atribuida a infecciones estafilocócicas, así como a medicamentos. La NET se asocia a estados de inmunosupresión. Sin embargo, se comprobó que la escaldadura estafilocócica y la NET eran entidades diferentes, con causas y fisiopatogenia distintas. Este padecimiento tiene una incidencia que oscila entre 0.93 y 1.3 casos por cada millón de habitantes por año. Es habitualmente causado por medicamentos; sin embargo, se han descrito casos tras vacunación contra sarampión-parotiditis-rubeola (triple viral), infección por Mycoplasma pneumoniae y por virus del dengue, tras reactivación de infección por citomegalovirus y posterior a la administración de agentes de contraste ${ }^{1,2}$.

Los medicamentos de riesgo alto confirmado que se encuentran asociados con este síndrome son los siguientes: nevirapina, lamotrigina, carbamazepina, fenitoína, fenobarbital, cotrimoxazol y otras sulfamidas, sulfasalazina, alopurinol, oxicam y otros antiinflamatorios no esteroideos. Así mismo, existen otros fármacos con riesgo potencial, tales como: paracetamol, ibuprofeno, pantoprazol y ácido acetilsalicílico, entre otros, pero que aún requieren de mayor evidencia. La patogenia consiste en una necrosis y desprendimiento de la epidermis debido a la apoptosis de queratinocitos inducida por un mecanismo inmunitario con un sustrato genético en ciertas poblaciones étnicas. Los principales inductores de la apoptosis de queratinocitos son las células T CD8+ y citotóxicas (como el Fas ligando [FasL]), junto con las células natural killer. La granulisina, una proteína citolítica que se encuentra dentro de estas células, desempeña un papel clave en la patogénesis².

Se han descrito diferentes factores de riesgo: sexo femenino, edad avanzada, pacientes con trastornos inmunológicos, cáncer, ascendencia asiática, predisposición familiar y portadores de HLA-B 1502 o HLA-A 3101. En pacientes con ascendencia asiática se recomienda evitar la carbamazepina ${ }^{3}$.

Las manifestaciones clínicas se presentan durante tres fases: periodo prodrómico, periodo de necrólisis y periodo de reepitelización. Durante el periodo prodrómico se presenta pérdida masiva de fluidos, el paciente puede presentar daño renal agudo, alteraciones electrolíticas (hiponatremia grave) y signos de hipoperfusión tisular (hipotensión, acidosis hiperlactatémica). Los síntomas iniciales generalmente se presentan 1-3 semanas después de la ingesta o aplicación del medicamento sospechoso. Algunos de los síntomas que se presentan son: fiebre, tos, rinorrea, conjuntivitis, anorexia y malestar general.

El periodo de necrólisis se caracteriza por un exantema macular doloroso de aparición súbita con sensación de dolor y quemazón que se extiende en un máximo de cuatro días; el exantema se distribuye simétricamente en la cara, parte superior del tronco y extremidades. Así mismo, el 90-95\% de los pacientes pueden presentar lesiones en mucosas tales como afectación ocular (fotofobia, dolor y pérdida de visión), afectación genitourinaria (retención urinaria, balanopostitis) y denudación completa de la mucosa gastrointestinal y respiratoria. El periodo de reepitelización se prolonga entre una y tres semanas en función de la extensión y gravedad. Las hiperpigmentaciones e hipopigmentaciones ocurren en prácticamente todos los pacientes; así mismo, también se presenta onicomadesis².

Para el diagnóstico se deberá verificar el tiempo transcurrido desde la ingesta del medicamento hasta la aparición del cuadro clínico. Para la valoración inicial se deberá evaluar si hay presencia de eosinofilia, linfocitosis con linfocitos atípicos, leucopenia, anemia normocítica, velocidad de eritrosedimentación globular aumentada, tiempo de protrombina y tromboplastina alargados, perfil hepático completo, hipoproteinemia, aumento de transaminasas, hipercolesterolemia, plaquetas, hemocultivos, cultivos de piel y orina y, si es necesario, de orificios corporales. De igual manera, las lesiones cutáneas que se presentan deberán ser evaluadas por la extensión del desprendimiento cutáneo de acuerdo con el signo de Nikolsky, el cual consiste en el desprendimiento de las capas superficiales de la epidermis al presionar lateralmente la piel aparentemente sana con la yema de los dedos (signo de Nikolsky positivo) ${ }^{4}$.

Entre las principales complicaciones de este síndrome se encuentran: desequilibrio hidroelectrolítico, infecciones bacterianas y micóticas en piel y mucosas, insuficiencia renal, edema agudo pulmonar, sepsis, choque séptico, falla multiorgánica y muerte.

Finalmente, el tratamiento médico se fundamenta en la retirada del fármaco causante y en el inicio de medidas de soporte. Estas últimas se pueden dividir en dos grupos según el efecto deseado: 
- Terapia inmunosupresora. Esta terapia consiste en el uso de ciclosporina $\mathrm{A}$ y corticosteroides sistémicos. La ciclosporina A, a dosis de $3 \mathrm{mg} / \mathrm{kg} /$ día durante 8-24 días, se asocia con rápida reepitelización. Así mismo, los corticosteroides sistémicos tienen propiedades antiinflamatorias, inmunosupresoras y antiapoptósicas, por los que son útiles en el tratamiento. Se han observado resultados benéficos con el uso de prednisona a dosis de $1-2 \mathrm{mg} / \mathrm{kg}$ durante tres a cinco días, y con dexametasona intravenosa a dosis de 1.5 $\mathrm{mg} / \mathrm{kg} /$ día durante tres días consecutivos.

- Medidas antiapoptósicas. La inmunoglobulina G (lgG) intravenosa inicialmente se propuso como tratamiento de la NET con base en el concepto de que el FasL es el principal mediador de la apoptosis de los queratinocitos ${ }^{5}$. En la NET es una indicación absoluta en la fase de agudización de los síntomas. La dosis es de $2 \mathrm{~g} / \mathrm{kg}$ en una sola aplicación o repartida en cinco días. A dosis altas en la fase clínica temprana reduce la mortalidad; el mecanismo propuesto es el bloqueo del CD95, promoviendo la supervivencia celular. Neutraliza, acelera el catabolismo y suprime la producción de anticuerpos, inhibe las moléculas de adhesión, modula la proliferación celular de células T y la apoptosis, y restaura el equilibrio $\mathrm{TH} 1-\mathrm{TH}_{2}{ }^{6}$.

Otra opción terapéutica es la plasmaféresis, con reporte de resultados exitosos de hasta un $80 \%$ en NET severa sin respuesta a ningún medicamento. El mecanismo de acción probable consiste en la eliminación del FasL. Su uso se basa en el principio de la eliminación de los fármacos, sus metabolitos y los mediadores citotóxicos de la sangre 5 .

\section{PRESENTACIÓN DEL CASO}

Mujer de 19 años de edad, peso $63 \mathrm{~kg}$ y talla de $150 \mathrm{~cm}$, residente del Estado de Tlaxcala. Con antecedentes de síndrome de Turner, hipotiroidismo diagnosticado hace dos meses y trastorno conductual caracterizado por desobediencia, falta de atención, irritabilidad y retraso en el aprendizaje, sin antecedentes de alergias conocidas, quirúrgicos, traumáticos ni transfusionales, sin otras enfermedades de relevancia y sin datos de hospitalización previa.

Paciente que inicia padecimiento el día 04/04/2018 con cuadro clínico de infección de vías respiratorias altas, por lo que acude a médico particular, quien diagnostica

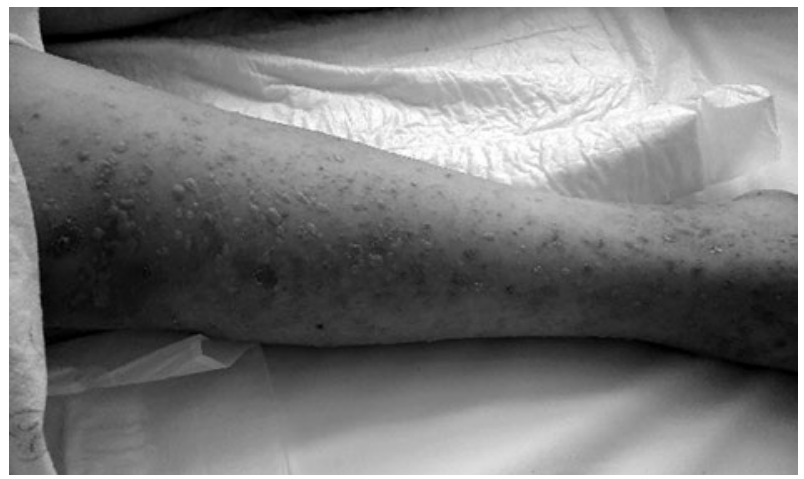

Figura 1. Miembro inferior (imagen propia, Hospital Regional de Alta Especialidad de Ixtapaluca).

faringoamigdalitis. Inicia tratamiento con ciprofloxacino $500 \mathrm{mg} / \mathrm{vo} / 8 \mathrm{~h}$, ibuprofeno $600 \mathrm{mg} / \mathrm{vo} / 8 \mathrm{~h}$, loratadina 10 mg/vo/24 r y carbamazepina 200 mg/vo/24 h; así mismo, se le prescribe tratamiento con levotiroxina $50 \mathrm{mcg} /$ vo/24 h. Posteriormente, el día 05/04/2018 comienza con lesiones dérmicas en región facial de color rojizo, acudiendo nuevamente al médico particular, quien incrementa la dosis de ciprofloxacino a 500 mg/6 h. Continúa con la presencia de lesiones eritematosas, por lo que acude al Hospital General de Tlaxcala el día 08/04/2018, de donde es referida al Hospital Regional de Alta Especialidad de Ixtapaluca (HRAEI) con diagnóstico de NET.

Ingresa a urgencias el 12/04/2018 presentando dermatosis diseminada en el $70 \%$ del cuerpo, constituida por múltiples lesiones de tipo vesículas y máculas diseminadas en cara, cuello, tórax, abdomen, extremidades superiores e inferiores, incluyendo manos y pies (Figs. 1-3), y genitales. En cavidad oral presenta placas necróticas con tejido friable en mucosa y región peribucal; en tórax posterior con placa de ámpulas confluentes que abarca toda la superficie de tórax posterior y región lumbar, además de genitales con mismas características, con signo de Nikolsky positivo, con edema importante en manos y plantas de pies. Mantiene una saturación de oxígeno del 96\%, se encuentran áreas pulmonares con hipoventilación basal bilateral, sin agregados, con movimientos ventilatorios conservados, afebril, sin datos de infección en lesiones dérmicas.

El 13/04/2018 se encuentra con tensión arterial $167 / 94 \mathrm{mmHg}$, tensión arterial media $118 \mathrm{mmHg}$, frecuencia cardiaca 57 Ipm y continúa con lesiones dérmicas en tórax, cara anterior y posterior, con máculas eritematosas purpúricas y edema. Presenta hiperglucemia $172 \mathrm{mg} / \mathrm{dl}$; la función hepática se encuentra sin 


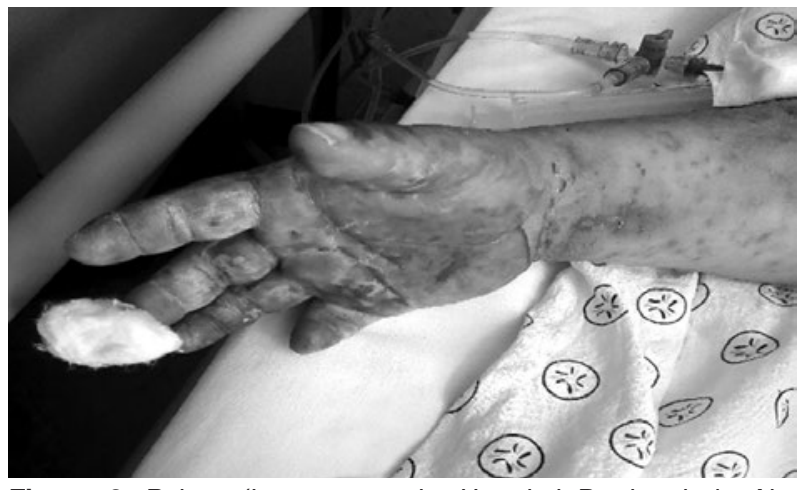

Figura 2. Palma (imagen propia, Hospital Regional de Alta Especialidad de Ixtapaluca).

alteraciones. Se retiran los medicamentos sospechosos y se inicia tratamiento a base de lgG $72 \mathrm{~g} / \mathrm{iv} / 24 \mathrm{~h}$, como analgésico tramadol $300 \mathrm{mg} / \mathrm{iv} / 24 \mathrm{~h}$, omeprazol $40 \mathrm{mg} /$ iv/24 h y metilprednisolona $500 \mathrm{mg} / \mathrm{iv} / 8 \mathrm{~h}$.

El 15/04/2018 se reportan cultivos negativos; continúa con lesiones dérmicas. El 16/04/2018 debido a retención de líquidos se agrega seroalbúmina $50 \mathrm{ml} / \mathrm{iv} / 8 \mathrm{~h}$ y furosemida $20 \mathrm{mg} / \mathrm{iv} / 8 \mathrm{~h}$.

El 17/04/2018 se encuentra sin deterioro neurológico; a nivel respiratorio, manteniendo ventilación espontánea; a nivel hemodinámico, presenta desequilibrio hidroelectrolítico (Tabla 1): hipocalcemia, hipomagnesemia, hiponatremia, hiperfosfatemia e hipocloremia. Se inició reposición hidroelectrolítica con gluconato de calcio 1 g/iv/8 h.

El 18/04/2018 en la cara se presentan costras meliséricas y hemáticas, con base eritematosa, que abarcan prácticamente la totalidad de la superficie de la piel de la cara; labios con presencia de costras hemáticas en toda su superficie, adheridas, que al desprenderse dejan base eritematosa con sangrado en capa. Se observan cambios de coloración con presencia de costras de color café, sin base eritematosa, con compromiso del $20 \%$ de superficie anterior de abdomen. Extremidades con presencia de costras meliséricas de predominio en dorso de las cuatro extremidades, con aumento del número de lesiones en región de dorsos, plantas y palmas, incluso con región de coloración negra en dedo anular de la mano derecha. Así mismo, inicia reactivación de las lesiones cutáneas, apareciendo nuevas vesículas de forma generalizada.

El 19/04/2018 la paciente continúa con hipocalcemia, teniendo un intervalo QT corregido de electrocardiograma

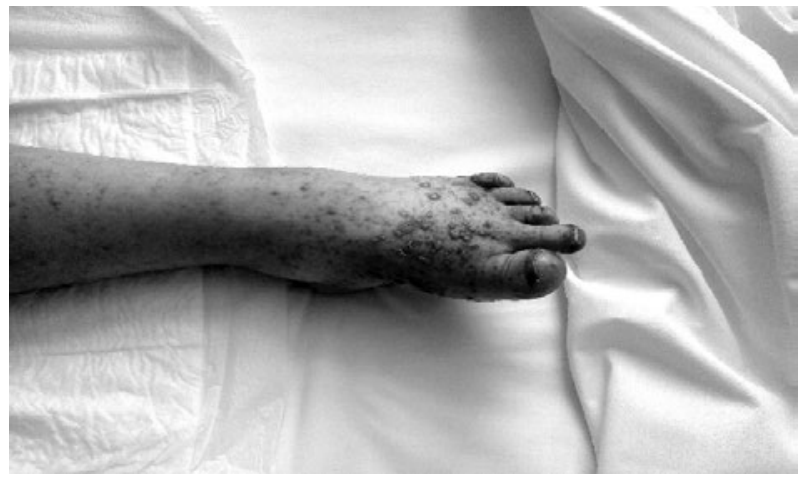

Figura 3. Dorso pie (imagen propia, Hospital Regional de Alta Especialidad de Ixtapaluca).

Tabla 1. Niveles de electrolitos séricos de la paciente el 17/04/2018

\begin{tabular}{lcc}
\hline Electrólito & Resultado & Valor de referencia \\
\hline Sodio & $132 \mathrm{meq} / \mathrm{l}$ & $136-145 \mathrm{meq} / \mathrm{l}$ \\
Cloro & $96 \mathrm{meq} / \mathrm{l}$ & $100-112 \mathrm{meq} / \mathrm{l}$ \\
Calcio & $7 \mathrm{mg} / \mathrm{dl}$ & $8.6-10.3 \mathrm{mg} / \mathrm{dl}$ \\
Fosforo & $5.6 \mathrm{mg} / \mathrm{dl}$ & $2.5-5 \mathrm{mg} / \mathrm{dl}$ \\
Magnesio & $1.6 \mathrm{mg} / \mathrm{dl}$ & $1.7-2.4 \mathrm{mg} / \mathrm{dl}$ \\
\hline
\end{tabular}

de 590 ms. Se indica manejo con infusión intravenosa de gluconato de calcio $11 \mathrm{~g}$ en 1,000 ml de solución glucosada al $5 \%$, con una velocidad de $50 \mathrm{ml} / \mathrm{h}$.

El 20/04/2018 se encuentra sin datos de infección activa dérmica, urinaria ni pulmonar. Se toman controles de electrólitos séricos, encontrando todos dentro de valores de referencia e intervalo QT corregido.

Para el 21/04/2018, sin aparición de nuevas vesículas, se realiza plasmaféresis. Para el 24/04/2018 la paciente presenta remisión de las lesiones y sin aparición de nuevas vesículas.

El 27/04/2018 se da de alta por mejoría, con piel reepitelizada, xerosis, manchas postinflamatorias residuales y electrólitos séricos corregidos.

\section{DISCUSIÓN}

En este reporte se aborda el caso de una paciente con diagnóstico de síndrome de Lyell. Presentó un cuadro agravado con descompensación electrolítica, principalmente hipocalcemia, hecho que se veía reflejado en la prolongación del intervalo QT, alcanzando un valor de 590 ms 
(siendo el valor normal entre 350 y $440 \mathrm{~ms}$ ), en el que ya se veía comprometida la función cardiaca, produciendo arritmias ventriculares.

En este caso, a pesar de que tanto la carbamazepina como el ciprofloxacino presentan riesgo potencial de producir NET, esta complicación tiene mayor incidencia con carbamazepina que con ciprofloxacino, específicamente en la población asiática, la cual presenta una variante alélica heredada, el HLA-B 1502 o HLA-A 3101.

Además, existe una interacción mayor entre la carbamazepina y la loratadina y una de menor riesgo entre el ciprofloxacino y la carbamazepina. En ambos casos se inhibe la isoenzima hepática CYP3A4, lo que provoca la disminución del metabolismo de la carbamazepina y, por ende, el aumento de su concentración en sangre.

La cuantificación de la concentración en suero de los medicamentos sospechosos no fue posible durante su administración concomitante debido a que esto sucedió antes de su ingreso al hospital. Sin embargo, al ingresar al HRAEI, mediante la revisión del expediente clínico de la paciente y a partir del trabajo multidisciplinario entre médicos y área de farmacovigilancia, se determinó que podía tratarse de un caso de NET atribuible al uso de carbamazepina y ciprofloxacino. Esto fue debido a que a la paciente se le estuvo administrando ciprofloxacino $500 \mathrm{mg} / \mathrm{vo} / 6 \mathrm{~h}$, dosis mayor a la recomendada para el tratamiento de neumonía, que es 250-750 mg/vo/12 h; al exceder la dosis, se potenció la aparición del síndrome de Lyell. La paciente fue tratada con Ig, metilprednisolona y plasmaféresis. Esta última ha reportado resultados exitosos de hasta el $80 \%$ en el síndrome de Lyell severo en el que no haya habido respuesta a algún medicamento ${ }^{5}$.

Aunque existen diversos tratamientos para el síndrome de Lyell, la base de evidencia es limitada y principalmente se enfoca a cuidados paliativos para el manejo del dolor y evitar infecciones de la piel. El tratamiento farmacológico es variable; a menudo se usan corticosteroides sistémicos, pero los estudios no han encontrado beneficio consistente ${ }^{7}$. Por otro lado, la Ig endovenosa parece mejorar la supervivencia, especialmente a dosis de $2 \mathrm{~g} / \mathrm{kg}^{8,9}$. En este caso en particular se observó una evolución favorable al tratamiento, en el cual la paciente ya presentaba desequilibrio electrolítico y alteración en el intervalo QT, por lo que se realizó manejo con aporte de calcio, observándose una evolución favorable.
El caso fue notificado por parte del médico tratante al personal del Centro Institucional de Farmacovigilancia del hospital el 16/04/2018, el cual realizó la investigación y análisis del caso y reportó al Centro Nacional de Farmacovigilancia.

\section{FINANCIAMIENTO}

La presente investigación no ha recibido ninguna beca específica de agencias de los sectores público, comercial o sin ánimo de lucro.

\section{CONFLICTO DE INTERESES}

Los autores declaran no tener conflicto de intereses.

\section{RESPONSABILIDADES ÉTICAS}

Protección de personas y animales. Los autores declaran que para esta investigación no se han realizado experimentos en seres humanos ni en animales.

Confidencialidad de los datos. Los autores declaran que han seguido los protocolos de su centro de trabajo sobre la publicación de datos de pacientes.

Derecho a la privacidad y consentimiento informado. Los autores han obtenido el consentimiento informado de los pacientes y/o sujetos referidos en el artículo.

\section{BIBLIOGRAFÍA}

1. Hötzenecker W, Prins C, French LE. Erythema multiforme, Stevens-Johnson syndrome, and toxic epidermal necrolysis. Dermatology. 2012;20:332-47.

2. Cachafeiro L, Sánchez MS, Lorente JA. Necrólisis epidérmica tóxica: un paradigma de enfermedad crítica. Rev Bras Ter Intensiva. 2017:29(4):499-508.

3. Guía de práctica clínica. Diagnóstico y tratamiento del síndrome de StevensJohnson/necrólisis epidérmica tóxica en el adulto [Internet]. México: Gobierno Federal, Secretaría de Salud; 2009. Disponible en: http://sgm.issste. gob mx/medica/medicadocumentacion/guiasautorizadas/ Reumatolog $\%$ C3 $\%$ ADa $\% 20$ e $\% 20$ Inmunolog $\%$ C3 $\%$ ACa/IMSS-398-10\%20 Diagn \%C3\%B3stico\%20y\%20tratamiento \%20de \%20SINDROME $\% 20$ DE\%20STEVENS\%20JOHNSON/398-10ER.PDF

4. Dabiri G, Rüenger T. Toxic epidermal necrolysis. En: Ferri FF. Ferri's Clinical Advisor 2018. 1st edition. Elsevier; 2018. pp. 1282-1282.

5. Pradilla Torres MT, Olmos E. Reacciones medicamentosas severas. Act Med Col. 2013;28(2):76-82.

6. Dolores M. Uso terapéutico actual de la inmunoglobulina intravenosa. Rev Med Inst Mex Seguro Soc. 2006;4(2):81-6.

7. Harr T, French LE. Toxic epidermal necrolysis and Stevens-Johnson syndrome. Orphanet J Rare Dis. 2010;5:39.

8. Roujeau JC, Bastuji-Garin S. Systematic review of treatments for StevensJohnson syndrome and toxic epidermal necrolysis using the SCORTEN score as a tool for evaluating mortality. Ther Adv Drug Saf. 2011;2(3):87-94

9. Barron SJ, Del Vecchio MT, Aronoff SC. Intravenous immunoglobulin in the treatment of Stevens-Johnson syndrome and toxic epidermal necrolysis: a meta-analysis with meta-regression of observational studies. Int J Dermatol. $2015 ; 54(1): 108-15$. 